\title{
Clinical Profile of Prosthetic Valve Endocarditis due to Candida parapsilosis: An 11-year Retrospective Observational Study from a Quaternary Cardiac Referral Institute in India
}

\author{
Dinoop K Ponnambath ${ }^{1} \odot$, Arun Gopalakrishnan ${ }^{2}$, Vivek V Pillai ${ }^{3} \odot$, Jyothi E Kaviyil ${ }^{4} \odot$, Kavita Raja $^{5} \odot$
}

\begin{abstract}
Background: Recent changes in the diagnostic criteria and the introduction of newer technologies like prosthetic valve replacement require the need to identify the changing epidemiology of prosthetic valve endocarditis (PVE).

Materials and methods: This is a retrospective, cross-sectional, observational study. Patients diagnosed with Candida parapsilosis definite and possible PVE as per modified Duke's criteria for a period of 11 years from January 2010 to December 2020 were included for the analysis. Results: Twelve of the 47 PVE cases (25.5\%) were caused by C. parapsilosis. The median age of the patients was 52 years. Males were predominantly affected (58\%). Based on the modified Duke's criteria, eight (67\%) were definite infective endocarditis (IE) cases. The single valve was affected in 11 cases $(92 \%)$ with the mitral valve being the commonest $(n=8,67 \%)$. The type of valve commonly involved was mechanical $[n=10,83 \%]$. The mean size of the vegetation was $13.15 \mathrm{~mm}$. Most cases $(n=7,58 \%)$ were late-onset PVE. The mean C-reactive protein (CRP), erythrocyte sedimentation rate (ESR), and procalcitonin (PCT) levels for C. parapsilosis PVE were $70.2 \mathrm{mg} / \mathrm{L}, 51.08 \mathrm{~mm} / \mathrm{hour}$, and $0.3 \mathrm{ng} / \mathrm{mL}$, respectively. The rates of complications and in-hospital mortality were $75 \%$ each. The most common observed complication was embolic events ( $n=8,67 \%$ ). Statistical significance $(p \leq 0.05)$ was observed for mean vegetation size, overall complications, embolic events, and mortality for $C$. parapsilosis PVE when compared with bacterial PVE.

Conclusion: C. parapsilosis was the commonest etiological agent causing PVE. Predominant mitral valve involvement, higher rates of late-onset presentation, complications, and mortality were key differential characteristics observed.

Keywords: Candida parapsilosis, Heart valve prosthesis, Infective endocarditis, Retrospective study.

Highlights: The manuscript throws light on the changing epidemiology, clinical, and microbiological profile of PVE due to Candida sp., which are scarcely studied and reported in low- and middle-income countries like India.

Indian Journal of Critical Care Medicine (2021): 10.5005/jp-journals-10071-23915
\end{abstract}

\section{INTRODUCTION}

Infective endocarditis (IE) is a relatively rare disease affecting the endothelial surface of the heart. The diagnosis is arrived using a combination of clinical, microbiological, and imaging findings guided by the modified Duke's criteria. Early identification ensures the institution of appropriate treatment leading to better clinical outcomes and lower risk of mortality. Recent changes in the diagnostic criteria and the introduction of newer technologies like prosthetic valve replacement require the need to identify the changing epidemiology of prosthetic valve endocarditis (PVE). PVE is a serious complication occurring after valve replacement surgery and is a distinct clinical entity when compared to native-valve endocarditis (NVE). The global annual incidence of PVE ranges between $1.4 \%$ and $3.1 \%$. $^{1}$ PVE is estimated to occur in 1-6\% of individuals undergoing prosthetic valve replacement surgery and approximately accounts for $10-30 \%$ cases of IE. ${ }^{2,3}$ The incidence of IE among patients with prosthetic valve is $6 / 1,000$ person years $(95 \% \mathrm{Cl}: 5.5-6.5 / 1,000$ person years), and the cumulative risk of developing PVE in patients with prosthetic valve is 2.8 and $4.5 \%$ at 5 and 10 years, respectively. ${ }^{4}$ The information on the epidemiology, clinical, and microbiological profile of PVE is scarce, particularly in low- and middle-income countries like India. This retrospective review describes the clinical profile of PVE caused by Candida parapsilosis.

\begin{abstract}
1,4,5 Department of Microbiology, Sree Chitra Tirunal Institute for Medical Sciences and Technology, Thiruvananthapuram, Kerala, India ${ }^{2}$ Department of Cardiology, Sree Chitra Tirunal Institute for Medical Sciences and Technology, Thiruvananthapuram, Kerala, India

${ }^{3}$ Department of Cardiovascular and Thoracic Surgery, Sree Chitra Tirunal Institute for Medical Sciences and Technology, Thiruvananthapuram, Kerala, India

Corresponding Author: Kavita Raja, Department of Microbiology, Sree Chitra Tirunal Institute for Medical Sciences and Technology, Thiruvananthapuram, Kerala, India, Phone: +91 9847400913, e-mail: kavita_raja@sctimst.ac.in

How to cite this article: Ponnambath DK, Gopalakrishnan A, Pillai VV, Kaviyil JE, Raja K. Clinical Profile of Prosthetic Valve Endocarditis due to Candida parapsilosis: An 11-year Retrospective Observational Study from a Quaternary Cardiac Referral Institute in India. Indian J Crit Care Med 2021;25(8):860-865.
\end{abstract}

Source of support: Nil

Conflict of interest: None

\section{Materials and Methods}

Aim: To analyze the clinical profile and outcome of PVE caused by $C$. parapsilosis. 
Study design: This is a retrospective, cross-sectional, observational study.

Study setting: The study was conducted at the Sree Chitra Tirunal Institute for Medical Sciences and Technology (SCTIMST), a 253-bedded quaternary referral center for cardiac and neurological disorders in Thiruvananthapuram, Kerala, South India.

Study approval: The study was approved by the Institute's Technical Advisory Committee (TAC) [SCT/S/2019/987 dated 25.09.019] and Institutional Ethics Committee (IEC) [SCT/IEC/1486/ November-2019]. The study period was from January 2010 to December 2020 (11 years).

Evaluation and diagnosis were based on clinical, echocardiographic, and microbiological criteria as per the modified Duke's criteria (Tables 1 and 2) proposed by the American Heart Association Scientific Statement, 2015. ${ }^{5}$

Inclusion criteria: All patients with prosthetic valve admitted with clinical suspicion of IE and diagnosed with definite and possible PVE due to Candida sp. as per modified Duke's criteria during the study period were included for the analysis.

Exclusion criteria: Patients with IE involving the native valve, cardiac device-related IE, that is, IE associated with implantable cardiac electronic devices and permanent pacemakers, infected intracardiac surgical baffles or conduits, and PVE caused by other etiological agents were excluded from the analysis.

Methodology: All patients underwent a transthoracic echocardiography (TTE) at admission, and patients with initial negative TTE but with a high index of IE suspicion subsequently underwent the transesophageal echocardiography (TEE) during the inpatient stay.

Three sets [BACT/ALERT FA (aerobic), and FN (anaerobic)] of blood cultures were collected with each set from different venipuncture sites, with the first and the last set collected at least 1 hour apart. The blood culture bottles were incubated in the automated continuous blood culture monitoring system (BACT/ ALERT 3D, bioMerieux, Inc, Durham, N.C). The yeast observed in positive blood culture bottles was isolated in pure culture on blood agar and Sabouraud dextrose agar. The identification of the yeast and further antifungal susceptibility was determined by estimation of minimum inhibitory concentration (MIC) for amphotericin $B$, flucytosine, fluconazole, voriconazole, caspofungin, and micafungin using the VITEK 2 YST ID and AST cards (bioMerieux, Marcy l'Etoile, France), respectively. The patient's data were gathered using a data collection tool, from the electronic medical records and laboratory information system of SCTIMST. Statistical analysis was performed in Microsoft Excel for Mac, version 16.46-2021, with the descriptive statistics expressed as frequencies and percentages for categorical variables and the median and interquartile range for continuous

Table 1: Definition of IE according to modified Duke's criteria

\begin{tabular}{ll}
\hline Classification category & Definition \\
\hline Definite IE & $\begin{array}{l}\text { Pathological criteria: Microorganisms demonstrated by culture or histological examination of a vegetation, a vege- } \\
\text { tation that has embolized, or an intracardiac abscess specimen; or pathological lesions; vegetation or intracardiac } \\
\text { abscess confirmed by histological examination showing active endocarditis } \\
\text { Clinical criteria: two major criteria, one major criterion and three minor criteria, or five minor criteria (Table 2) } \\
\text { Possible IE }\end{array} \quad \begin{array}{l}\text { One major criterion and one minor criterion, or three minor criteria } \\
\text { Rejected IE }\end{array}$ \\
$\begin{array}{l}\text { Firm alternative diagnosis explaining evidence of IE; or resolution of IE syndrome with antibiotic therapy for } \leq 4 \\
\text { days; or no pathological evidence of IE at surgery or autopsy with antibiotic therapy for } \leq 4 \text { days; or does not meet } \\
\text { criteria for possible IE as above }\end{array}$ \\
\hline
\end{tabular}

Table 2: Definition of terms used in the clinical criteria of modified Duke's criteria for the diagnosis of IE

\begin{tabular}{ll}
\hline Clinical criteria & Definition \\
\hline Major & 1. Blood culture positive for IE: Typical microorganisms consistent with IE from two separate blood cultures: \\
& Viridans streptococci, Streptococcus bovis, HACEK group, Staphylococcus aureus; or community-acquired enterococci in \\
the absence of a primary focus, or microorganisms consistent with IE from persistently positive blood cultures defined as \\
follows: at least two positive cultures of blood samples drawn $>12$ hours apart or all three or a majority of $\geq 4$ separate \\
cultures of blood (with first and last sample drawn at least 1 hour apart) \\
Single-positive blood culture for Coxiella burnetii or antiphase 1 IgG antibody titer $\geq 1: 800$ \\
2. Evidence of endocardial involvement: \\
Echocardiogram positive for IE [TEE recommended for patients with prosthetic valves, rated at least possible IE by \\
clinical criteria, or complicated IE (paravalvular abscess); TTE as first test in other patients] defined as follows: Oscillating \\
intracardiac mass on valve or supporting structures, in the path of regurgitant jets, or on implanted material in the \\
absence of an alternative anatomic explanation; abscess; or new partial dehiscence of prosthetic valve or new valvular \\
regurgitation (worsening or changing or preexisting murmur not sufficient) \\
1. Predisposition, predisposing heart condition, or IDU \\
2. Fever, temperature $>38^{\circ} \mathrm{C}$ \\
3. Vascular phenomena, major arterial emboli, septic pulmonary infarcts, mycotic aneurysm, intracranial hemorrhage, \\
conjunctival hemorrhage, and Janeway lesions \\
4. Immunological phenomena: Glomerulonephritis, Osler's nodes, Roth spots, and rheumatoid factor \\
5. Microbiological evidence: Positive blood culture but does not meet a major criterion as noted above (excludes single- \\
positive cultures for CoNS and organisms that do not cause endocarditis) or serological evidence of active infection with \\
organism consistent with IE
\end{tabular}


variables. Statistical analysis was done using IBM statistical package for the social sciences statistics 25.0. The categorical variables were compared using a two-tailed Chi-square test, and a $p$-value of $\leq 0.05$ was considered statistically significant.

\section{Results}

A total of 47 cases of PVE were diagnosed during the study period. Thirteen of the 47 cases (27.6\%) (Fig. 1) were caused by Candida sp., of which 12 cases (25.6\%) were caused by C. parapsilosis and a single case (2\%) by Candida haemulonii. Based on the modified Duke's criteria, eight (67\%) were definite and four (33\%) were possible IE cases.

The median age of the patients diagnosed with PVE due to $C$. parapsilosis was 52 years [interquartile range (IQR): 43-55]. Males were predominantly affected $(n=7,58 \%)$ in this study. The median time between prosthetic valve implantation and diagnosis of PVE was 26 months [IQR: 9.25-64.75 months]. Late-onset cases were observed in seven cases (58\%), while the remaining five cases (42\%) were of early-onset.

Valve affected: Single valve was affected in 11 cases (92\%), while double valve involvement was noticed in a single case (8\%). Mitral valve was affected in eight cases $(67 \%)$, while the aortic valve was affected in five cases (42\%). There were no cases of involvement of right-sided prosthetic heart valves. Among the type of valve, the mechanical prosthetic valve was involved in 10 cases (83\%), and bioprosthetic (homograft) was involved in 2 cases (17\%). The size of the vegetation determined based on TTE/TEE was large $(\geq 10 \mathrm{~mm})$ in eight cases (67\%), while moderate-sized (5-10 mm) in one case (8\%) and small-sized $(<5 \mathrm{~mm})$ in another case $(8 \%)$. The mean size of the vegetation was large $(13.15 \mathrm{~mm}$, range: $8 \mathrm{~mm})$ for PVE caused by $C$. parapsilosis, while it was moderate-sized $(7.35 \mathrm{~mm}$, range: $15 \mathrm{~mm})$ in the bacterial PVE group.

The mean C-reactive protein (CRP) and procalcitonin (PCT) levels observed in patients with C. parapsilosis PVE were $70.2 \mathrm{mg} / \mathrm{L}$ and $0.3 \mathrm{ng} / \mathrm{mL}$, respectively. In three C. parapsilosis PVE cases, the CRP levels were greater than $100 \mathrm{mg} / \mathrm{L}$. The mean ESR level observed in this cohort was $51.08 \mathrm{~mm} /$ hour. The rate of complications at presentation was $75 \%(n=9)$. The most commonly observed complication was an embolic event $(n=8$, $67 \%)$. The embolic events occurred in the following sites: central

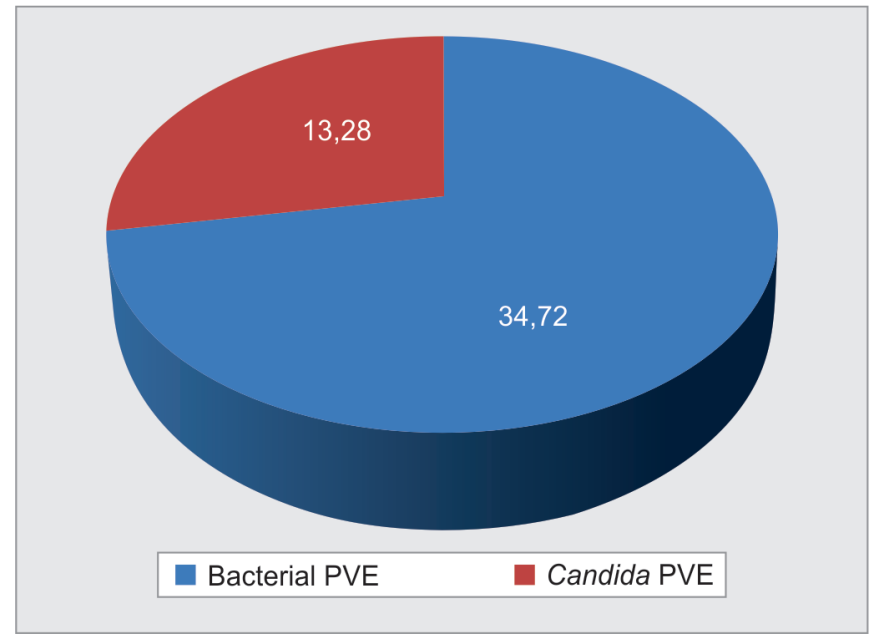

Fig. 1: Proportion of PVE caused by Candida and bacteria $(n, \%)$ nervous system ( $n=4,50 \%)$, spleen ( $n=3,38 \%)$, lower limb ( $n=2$, $25 \%)$, and kidney $(n=1,13 \%)$.The other complications observed were paravalvular abscess $(n=2,17 \%)$, intracranial bleed $(n=2$, $17 \%)$, and renal failure ( $n=2,17 \%)$ usually secondary to the use of liposomal amphotericin B (LAMB).

Ten cases (83\%) were managed with LAMB-based combination antifungal therapy with one case managed with additional surgical intervention, while LAMB monotherapy was used in two cases (17\%). The regimens used for combination therapy were LAMB with either flucytosine $(n=2,17 \%)$ or azoles [fluconazole $(n=5,42 \%)$ or voriconazole $(n=3,25 \%)$ ]. Echinocandins [caspofungin $(n=1)$ or micafungin $(n=1)$ ] were included in the regimen as a replacement for LAMB when renal toxicity was observed during the course of 6-week therapy. The in-hospital mortality rate observed in this cohort of PVE due to C. parapsilosis was $75 \%(n=9)$.

The clinical characteristics, complications, and outcome between bacterial and C. parapsilosis PVE were compared, and the results are shown in Table 3. Statistical significance was observed for mean vegetation size $(p<0.03)$, overall frequency of complications ( $p<0.00001)$, embolic events $(p<0.00001)$, and mortality $(p<0.0006)$ for $C$. parapsilosis PVE when compared to bacterial PVE group.

\section{Discussion}

This retrospective observational study is the largest study from India describing the clinical profile and outcome of patients with PVE caused by C. parapsilosis. PVE is a serious complication following a cardiac valve replacement surgery. In the study by Pierrotti and Baddour (1965-1995), the median age of the patients with Candida IE was 44 years, a relatively younger population although intravenous drug use (IDU) was identified as a risk factor in only $4.1 \%$ of cases. ${ }^{6}$ In this study and other recent published studies, the median age of the patients with Candida PVE (PVE-C) was greater than 50 years [56.4 years (Giuliano et al.) and 66 years (Rivoisy et al.)]. ${ }^{7,8}$ This shows a trend of increasing median age in the occurrence of PVE-C over the past two decades.

The etiological agents causing PVE vary based on the duration of onset of IE after surgery and are classified as early(within 1 year of valve replacement) and late-onset (after 1 year of valve replacement). Early-onset PVE commonly caused by Staphylococcus aureus, coagulase-negative staphylococci (CoNS), Gram-negative bacilli, etc., occurs due to infection or contamination of the valve during the surgical procedure and the perioperative period, while the common etiological agents causing late PVE (Enterococcus, viridans group streptococci, etc.) are similar to NVE. Early studies over the past few decades show that Candida sp. was never reported as a common pathogen causing early- or late-onset PVE in spite of the widespread use of mechanical valves since the 1970 s. ${ }^{9,10}$ Most of the early-onset PVE occur within the first 3 months, and hence, few authors mention the ideal cutoff period as 120 days (4 months) for classification as early- and late-onset PVE. If this proposed cutoff period is chosen for classification, then all PVE caused by C. parapsilosis (100\%) in this study will be classified as lateonset PVE.

In a review of 152 cases of fungal endocarditis (1965-1995), Candida sp. was responsible for $94.1 \%$ of yeast endocarditis and Aspergillus sp. for $71.8 \%$ of mold endocarditis. ${ }^{6}$ The overall ratio between nonalbicans Candida and C. albicans IE was 1.2:1. In the Italian study on endocarditis (SEI), the commonest Candida species 
A Retrospective Clinical Profile Study of Candida parapsilosis PVE

Table 3: Comparison of clinical characteristics, complications, and outcome between bacterial and C. parapsilosis PVE

\begin{tabular}{|c|c|c|c|}
\hline Patient characteristics & Bacterial PVE & C. parapsilosis PVE & $p$-value \\
\hline A. Median age (years) & 53 & 52 & 0.99 \\
\hline B. Median time to presentation after valve replacement (months) & 34 & 26 & 0.36 \\
\hline \multicolumn{4}{|l|}{ C. Onset of presentation (\%) } \\
\hline Early PVE & 38 & 42 & 0.66 \\
\hline Late PVE & 62 & 58 & 0.66 \\
\hline \multicolumn{4}{|l|}{ D. Valve affected (\%) } \\
\hline \multicolumn{4}{|l|}{ Mitral valve } \\
\hline \multicolumn{4}{|l|}{ Aortic valve } \\
\hline E. Mean vegetation size $(\mathrm{mm})$ & 7.35 & 13.15 & 0.03 \\
\hline \multicolumn{4}{|l|}{ F. Laboratory parameters } \\
\hline Mean CRP level (mg/L) & 65.2 & 70.2 & 0.68 \\
\hline Mean ESR level (mm/hour) & 43.5 & 51.08 & 0.79 \\
\hline Mean PCT level (ng/mL) & 0.44 & 0.36 & 0.92 \\
\hline \multicolumn{4}{|l|}{ G. Complications (at presentation and during hospital stay) [\%] } \\
\hline Overall (total) & 26 & 74 & $<0.00001$ \\
\hline Embolic events & 18 & 67 & $<0.00001$ \\
\hline H. In-hospital mortality (\%) & 21 & 75 & 0.0006 \\
\hline
\end{tabular}

The bold values indicate statistically significant values $(<0.05)$ on comparison of two groups.

causing IE was C. albicans, while in the ESCAPE study, the ratio between nonalbicans Candida sp. and C. albicans IE was 1:1.7,8

Candida endocarditis has been described as a biofilm infection that can affect the prosthetic and native valves. The development of late-onset PVE-C is described to occur as a two-step process: (1) Candidemia occurring during surgery or early postoperative period or during subsequent hospital admissions leading to colonization of the prosthetic valve and (2) biofilm formation and progressive tissue invasion leading to clinical features of IE and/or detectable vegetations in cardiac imaging. Detection of episodes of candidemia in patients with prosthetic valves was identified as a strong predictor for the complication of late-onset PVE. ${ }^{11}$ The common risk factors for candidemia after open-heart surgery include prolonged cardiopulmonary bypass procedure (>120 minutes), mechanical ventilation $>10$ days, prolonged hospital or ICU admission (>20 days), diabetes mellitus, sepsis, broad-spectrum antibiotic use, central venous catheter use, total parenteral nutrition, renal injury, or patients on renal replacement therapy. ${ }^{12}$

TTE is used as the screening imaging technique for assessing the hemodynamics, mobility, and stability of the prosthetic valve. Detection of vegetations is difficult in TTE, particularly in the early stages of biofilms due to valve artifacts, and hence, the range of sensitivity of TTE for detecting vegetations in patients with prosthetic valve is very low (36-69\%). The use of TEE in patients with prosthetic valve increases the chance of detection of vegetations (sensitivity: 86-94\%) and hence definite IE in patients with prosthetic valve. ${ }^{13}$ Significant differences in the proportion of definite Candida IE case identification have been reported between the pre-TEE (53.3\%) and post-TEE (84.4\%) groups. ${ }^{7}$ The identification of definite PVE after utilizing TEE was observed in a single case in this study. In our study, TEE was not used in the diagnosis of all cases of IE, and three possible PVE cases did not have vegetations in TTE. Utilization of TEE for negative-TTE patients could have increased the diagnosis of definite IE cases but carries the risk of embolic events. The introduction of novel imaging techniques like ${ }^{18} \mathrm{~F}$-fluorodeoxyglucose positron emission tomography/computed tomography (FDG-PET/CT) after
3 months of surgery, leucocyte-labelled SPECT/CT, and cardiac CT can increase the chances of detecting vegetations due to their higher sensitivity in the detection of inflammatory foci in prosthetic valves. The finding of abnormal leucocyte-labelled SPECT/CT or FDG accumulation or paravalvular lesions in CT in and around the artificial valve has been proposed as a major criterion for the diagnosis of PVE. ${ }^{14,15}$ Implementation of these advanced imaging techniques can lead to increased diagnosis of PVE (definite IE) and early identification of perivalvular complications.

The aortic valve was affected in 72 and $64.1 \%$ of PVE-C cases in the ESCAPE and the Italian study on endocarditis (SEI), respectively. In contrast to the published literature, the mitral valve was the commonest valve affected in our cohort (67\%). Among the valve types, a bioprosthetic valve is recommended for individuals above 70 years due to its reduced durability, while the mechanical valve is durable (hence recommended in younger individuals) yet prone to the risk of thrombogenicity, therefore, the requirement of lifelong anticoagulants. ${ }^{16} \mathrm{~A}$ recent systematic review and metaanalysis showed that patients with bioprosthetic valves are at higher odds (1.59 times) of developing IE than patients with mechanical valves. ${ }^{17}$ An increase in late-onset PVE in our study could be due to the higher proportion of mechanical valves affected in our cohort (83\%). The endothelialization of the mechanical valve is a longprocess (an average of 3-10 months extending to 18-24 months) and hence the reason for the late presentation of PVE in patient with mechanical valves. ${ }^{18}$

The major risk factor for Candida IE observed in this study was the presence of a prosthetic valve (86.6\%). C. parapsilosis was the commonest species of Candida causing IE and PVE in our study, while in the study by Arnold et al. and in most other studies, $C$. albicans was the commonest species causing PVE. ${ }^{19}$ Candida sp. as an etiological agent for NVE was observed in three cases $(2-C$. parapsilosis; 1-C. pelliculosa) in this study. The most common clinical complication in their study was systemic embolization (34\%), followed by CHF (31\%) and intracardiac abscess (24\%). In the study by Rivoisy et al., $85 \%$ of cases were healthcare-related or nosocomial, and $20 \%$ were intravenous drug users. In the study 
by Arnold et al., ${ }^{19}$ majority of Candida IE were of nosocomial origin (67\%). The global mortality rate for PVE-C was estimated as $56 \%$ in the ESCAPE study. On comparison of the MYCENDO (NVE due to Candida sp.) and ESCAPE study (PVE due to Candida sp.), the 6-month cumulative mortality rate was higher in NVE-C (57\%), while $37 \%$ in PVE. 8,20

Patients who received LAMB alone had a better survival rate than those who received echinocandin alone. According to Center for Disease Control and the European Society of Clinical Microbiology and Infectious Diseases (ESCMID) guidelines, PVE-C should be managed with antifungals and early surgery, but surgery was not associated with better 6-month survival rate. A report by Soldini et al. ${ }^{21}$ found that C. parapsilosis isolated from device-associated infections [e.g., central venous catheter (CVC)-related bloodstream infections] was of high or moderate biofilm formation (HBF/ MBF) potential. Elevated MIC values for azoles and higher 30-day mortality when treated with azole antifungals were observed for device-associated blood-stream infections caused by HBF/MBF C. parapsilosis isolates. ${ }^{21}$ Initial recommendation by the Infectious Diseases Society of America (IDSA) was the use of maintenance therapy with fluconazole for patients who did not undergo surgery, while the 2016 updated guidelines recommend chronic suppressive antifungal therapy with 400 to $800 \mathrm{mg}$ fluconazole/day irrespective of whether patients undergo surgery, to prevent recurrences. Recently, IDSA and ESCMID have endorsed the use of echinocandins for the treatment of Candida IE. ${ }^{22,23}$ Similar to rifampicin use for the treatment for biofilm formation in S. aureus PVE, echinocandins and liposomal preparations of amphotericin $B$ have been found to be effective against Candida biofilms. ${ }^{24}$ Arnold et al. ${ }^{19}$ reported no difference in efficacy between echinocandin-based and amphotericin B-based antifungal regimens, while Giuliano et al. ${ }^{7}$ reported lower mortality odds when any of the antibiofilm agents were included. The regimens were classified as highly active antibiofilm if patients were started on echinocandin-based regimen and moderately active antibiofilm regimen if started on amphotericin B-based regimen. The therapeutic strategies reported to enhance survival were effective antibiofilm (EAB) induction treatment, chronic suppressive maintenance antifungal treatment, and cardiac surgery. Hence, treatment regimens recommended for PVE-C include two phases: Induction phase of 6 weeks with a high or moderate antibiofilm antifungal agent-amphotericin $B$ or caspofungin followed by a lifelong suppressive maintenance therapy with high-dose fluconazole. Longer prospective multicentric studies are required to prove the efficacy of such recommended regimens. Early surgical intervention should be considered in all cases, particularly for patients with vegetation size $>10 \mathrm{~mm}$, once candidemia clearance has been achieved.

\section{Conclusion}

C. parapsilosis was the commonest cause of PVE (25.6\%) in contrast to the global published literature (1.7-3.8\%). Predominant mitral valve involvement, higher median time to presentation, complications, and in-hospital mortality were key differential characteristics observed when compared with the literature review and analysis of PVE-C from the Italian study on endocarditis (SEI) and the ESCAPE study.

\section{ORCID}

Dinoop K Ponnambath @ (t) hs://orcid.org/0000-0002-0644-3908 Arun Gopalakrishnan (1) https://orcid.org/0000-0003-1350-8076
Vivek V Pillai @ https://orcid.org/0000-0002-8227-9919

Jyothi E Kaviyil @ i https://orcid.org/0000-0001-8381-8438

Kavita Raja 우 https://orcid.org/0000-0003-1914-5034

\section{References}

1. Siciliano RF, Randi BA, Gualandro DM, Sampaio RO, Bittencourt MS, da Silva Pelaes $C E$, et al. Early-onset prosthetic valve endocarditis definition revisited: Prospective study and literature review. Int J Infect Dis 2018;67:3-6. DOI: 10.1016/j.ijid.2017.09.004.

2. Luciani N, Mossuto E, Ricci D, Luciani M, Russo M, Salsano A, et al. Prosthetic valve endocarditis: predictors of early outcome of surgical therapy. a multicentric study. Eur J Cardio-Thoracic Surg 2017;52(4):768-774. DOI: 10.1093/ejcts/ezx169.

3. Ivanovic B, Trifunovic D, Matic S, Petrovic J, Sacic D, Tadic M. Prosthetic valve endocarditis - a trouble or a challenge? J Cardiol 2019;73(2):126133. DOI: 10.1016/j.jjcc.2018.08.007.

4. Østergaard L, Valeur N, Ihlemann N, Bundgaard H, Gislason G, TorpPedersen C, et al. Incidence of infective endocarditis among patients considered at high risk. Eur Heart J 2018;39(7):623-629. DOI: 10.1093/ eurheartj/ehx682.

5. Baddour LM, Wilson WR, Bayer AS, Fowler VG, Tleyjeh IM, Rybak MJ, et al. Infective endocarditis in adults: diagnosis, antimicrobial therapy, and management of complications: a scientific statement for healthcare professionals From the American Heart Association. Circulation 2015;132(15):1435-1486. DOI: 10.1161/ CIR.0000000000000296.

6. Pierrotti LC, Baddour LM. Fungal endocarditis, 1995-2000. Chest 2002;122(1):302-310. DOI: 10.1378/chest.122.1.302.

7. Giuliano S, Guastalegname M, Russo A, Falcone M, Ravasio V, Rizzi $M$, et al. Candida endocarditis: systematic literature review from 1997 to 2014 and analysis of 29 cases from the Italian Study of Endocarditis. Expert Rev Anti-infect Ther 2017;15(9):807-818. DOI: 10.1080/14787210.2017.1372749.

8. Rivoisy C, Vena A, Schaeffer L, Charlier C, Fontanet A, Delahaye F, et al. Prosthetic valve Candida spp. endocarditis: new insights into longterm prognosis—the ESCAPE study. ClinInfect Dis 2018;66(6):825832. DOI: $10.1093 / \mathrm{cid} / \mathrm{cix} 913$.

9. Ellis ME, Al-Abdely H, Sandridge A, Greer W, Ventura W. Fungal endocarditis: evidence in the world literature, 1965-1995. Clin Infect Dis 2001;32(1):50-62. DOI: 10.1086/317550.

10. Rivas $\mathrm{P}$, Alonso J, Moya J, de Górgolas M, Martinell J, Fernández Guerrero ML. The impact of hospital-acquired infections on the microbial etiology and prognosis of late-onset prosthetic valve endocarditis. Chest 2005;128(2):764-771. DOI: 10.1378/chest.128.2.764.

11. Russo A, Falcone M, Picciarella A, Giuliano S, Raponi G, Venditti M. Candidaemia after heart valve replacement surgery: recurrence as prosthetic valve endocarditis is an expected over one-year complication. Clin Microbiol Infect 2016;22(5):466-467. DOI: 10.1016/j. cmi.2016.01.019.

12. Giacobbe DR, Salsano A, Del Puente F, Miette A, Vena A, Corcione S, et al. Risk factors for candidemia after open heart surgery: results from a multicenter case-control study. Open Forum Infect Dis 2020;7(8):ofaa233. DOI: 10.1093/ofid/ofaa233.

13. Evangelista A, Gonzalez-Alujas MT. Echocardiography in infective endocarditis. Heart 2004;90(6):614-617. DOI: 10.1136/hrt.2003.029868.

14. Bruun NE, Habib G, Thuny F, Sogaard P. Cardiac imaging in infectious endocarditis. Eur Heart J 2014;35(10):624-632. DOI: 10.1093/eurheartj/ eht274.

15. Habib G, Lancellotti $P$, Antunes MJ, Bongiorni MG, Casalta J-P, Del Zotti F, et al. 2015 ESC Guidelines for the management of infective endocarditis: the task force for the Management of Infective Endocarditis of the European Society of Cardiology (ESC)Endorsed by: European Association for Cardio-Thoracic Surgery (EACTS), the European Association of Nuclear Medicine (EANM). Eur Heart J 2015;36(44):3075-3128. DOI: 10.1093/eurheartj/ehv319.

16. Nishimura RA, Otto $C M$, Bonow RO, Carabello BA, Erwin JP, Fleisher LA, et al. 2017 AHA/ACC Focused Update of the 2014 AHA/ 
ACC guideline for the management of patients with valvular heart disease: a report of the American College of Cardiology/American Heart Association Task Force on Clinical Practice Guidelines. Circulation [Internet] 2017;135(25):e1159-e1195. DOI: 10.1161/ CIR.0000000000000503.

17. Anantha-Narayanan M, Reddy YNV, Sundaram V, Murad MH, Erwin PJ, Baddour LM, et al. Endocarditis risk with bioprosthetic and mechanical valves: systematic review and meta-analysis. Heart 2020;106(18):1413-1419. DOI: 10.1136/heartjnl-2020-316718.

18. Nonaka M, Kusuhara T, An K, Nakatsuka D, Sekine Y, Iwakura A, et al. Comparison between early and late prosthetic valve endocarditis: clinical characteristics and outcomes. J Heart Valve Dis 2013;22(4):567-574. PMID: 24224422.

19. Arnold CJ, Johnson M, Bayer AS, Bradley S, Giannitsioti E, Miró JM, et al. Candida infective endocarditis: an observational cohort study with a focus on therapy. Antimicrob Agents Chemother 2015;59(4):2365-2373. DOI: 10.1128/AAC.04867-14.

20. Lefort A, Chartier L, Sendid B, Wolff M, Mainardi J-L, Podglajen I, et al. Diagnosis, management and outcome of Candida endocarditis.
Clin Microbiol Infect 2012;18(4):E99-E109. DOI: 10.1111/j.14690691.2012.03764.x.

21. Soldini S, Posteraro B, Vella A, De Carolis E, Borghi E, Falleni M, et al. Microbiologic and clinical characteristics of biofilm-forming Candida parapsilosis isolates associated with fungaemia and their impact on mortality. Clin Microbiol Infect 2018;24(7):771-777. DOI: 10.1016/j. cmi.2017.11.005.

22. Pappas PG, Kauffman CA, Andes DR, Clancy CJ, Marr KA, OstroskyZeichner $L$, et al. Clinical practice guideline for the management of candidiasis: 2016 update by the Infectious Diseases Society of America. Clin Infect Dis 2016;62(4):e1-e50. DOI: 10.1093/cid/civ933.

23. Cornely OA, Bassetti M, Calandra T, Garbino J, Kull lberg BJ, Lortholary O, et al. ESCMID* guideline for the diagnosis and management of Candida diseases 2012: non-neutropenic adult patients. Clin Microbiol Infect 2012;18:19-37. DOI: 10.1111/1469-0691.12039.

24. Høiby N, Bjarnsholt T, Moser C, Bassi GL, Coenye T, Donelli G, et al. ESCMID* guideline for the diagnosis and treatment of biofilm infections 2014. Clin Microbiol Infect 2015;21:S1-25. DOI: 10.1016/j. cmi.2014.10.024. 